\title{
Non-random mating and information theory
}

\author{
A. Carvajal-Rodríguez
}

Departamento de Bioquímica, Genética e Inmunología. Universidad de Vigo, 36310

Vigo, Spain.

*: A. Carvajal-Rodríguez. Departamento de Bioquímica, Genética e Inmunología.

Universidad de Vigo, 36310 Vigo, Spain. Phone: +34 986813828

email:acraaj@uvigo.es

Keywords: Mate choice, sexual selection, sexual isolation, Price equation, KullbackLeibler divergence, population genetics. 


\section{Abstract}

1 In this work, mate choice is modeled by means of the abstract concept of mutual mating

2 propensity. This only assumes that different type of couples can have different mating

3 success. The model is adequate for any population where mating occurs among distinct

4 types. There is no extra assumption about particular mating scheme or preference

5 model. The concept of mutual mating propensity permits to express the observed

6 change in the mating phenotypes as the gain in information with respect to random

7 mating. The obtained expression is a form of the Price equation in which the mapping

8 between ancestral and descendant population is substituted by a mapping between

9 random mating and non random mating population.

10 At the same time, this framework provides the connection between mate choice and the

11 exact mathematical partition of the choice effects, namely sexual isolation, sexual

12 selection and a mixed effect. The sexual selection component is the sum of the intra-

13 sexual male and female selection.

14 The proposed framework helps to unveil previously hidden invariants. For example, if

15 the mutual preference between partner types is multiplicative there is no sexual isolation

16 (inter-sexual selection) effect on the frequencies, i.e. the only possible effect of mate

17 choice is intra-sexual selection. On the contrary, whatever the contribution of each

18 partner to the mutual preference, if it comes as a non-multiplicative factor, there is at

19 least an inter-sexual selection detectable effect.

20 This new view over the mate choice problem, permits to develop general mating

21 propensity models and to make predictions of the mate choice effects that may emerge

22 from such models. This possibility opens up the way for setting a general theory of model fitting and multimodel inference for mate choice. 
24 Thus, it is suggested that the proposed framework, by describing mate choice as the

25 flow of information due to non-random mating, provides a new important setting for

26 exploring different mating models and their consequences. 


\section{Introduction}

Mate choice is arguably one of the most active areas of evolutionary research. There has

30 been a lot controversy regarding the concept of mate choice. The debate around mate

31 choice was due in part to its importance for fields so diverse as population genetics,

32 evolutionary-ecology, animal behavior, sociology, or psychology. In addition, there has

33 been an excess of verbal models and imprecise terminology regarding different aspects

34 of mate choice (Edward, 2015). Mate choice can be broadly described as the effect of

35 some expressed traits leading to non-random mating. Under this broad definition there

36 are various aspects that can be considered. Yet Darwin (1871) distinguishes between

37 intrasexual selection and intersexual selection. The first arises directly from competition

38 among individuals of the same sex while the second arises from choice of mates by the

39 other sex (Kuijper et al., 2012). Alternatively, from a population genetics point of view,

40 mate choice is defined as the observed mating frequency deviation with respect to

41 random mating, considering population gene or phenotype frequencies. So defined,

42 mate choice can be partitioned into (intra)sexual selection, defined as the observed

43 change in gene or phenotype frequencies in mated individuals with respect to

44 population frequencies, and sexual isolation (behavioral isolation or intersexual

45 selection), defined as the deviation from random mating in mated individuals (Rolán-

46 Alvarez and Caballero, 2000). In this work I followed these definitions of mate choice,

47 intrasexual and intersexual selection.

For an alternative description of these concepts and a discussion about some of the most

49 widely used descriptions of evolutionary change within the context of sexual selection, I

50 refer the reader to (Kuijper et al., 2012; Rosenthal, 2017). 
51 The many aspects and complexity of mate choice justifies the extensive research that

52 has been made in the last decades producing several theoretical models and empirical tests. Related to modeling and detection of mate choice, there is the question about the correct null hypothesis for testing the evolution of mate choice. The Lande-Kirpatrick (L-K) model has been proposed as a null model (Kirkpatrick, 1982; Lande, 1981; Prum, 2010; Roff and Fairbairn, 2014). This model assumes neutral genetic variation for the mating preference trait while the target trait can be under natural selection. However, the L-K role as a null model is not clear when the preference is set by similarity (preference and target trait coincide) and the trait is under divergent selection (Servedio

60 et al., 2011), i.e. the trait is "magic" sensu Gavrilets (2004), because in this case the 61 preference trait is already under selection (Hughes, 2015).

62 Therefore, there is still a need for both, null models and a general framework, where the 63 key essential facts of the mate choice can be adequately described. Here, I argue that the 64 formalism provided by the information theory in the form of the Jeffreys' divergence is 65 the right tool to do so.

66 The information theory has already been elegantly applied for describing evolutionary

67 change (Frank, 2009; Frank, 2012b; Frank, 2013). The present work takes advantage of that mathematical structure and applies it for modeling the change in mating frequencies

69 due to mate choice. As far as I know there is no previous attempt of describing mate

70 choice from the viewpoint of the information theory. Nevertheless, the potential of the 71 informational view for evolutionary ecology has been already suggested (Dall et al., 72 2005).

73 First, I defined a general model that only requires an abstract functional relationship 74 connecting the observed mating frequencies with the expected by random mating from 
75 the population gene or phenotype frequencies. This suffices for developing a general

76 information equation for mate choice that can be adequately partitioned into intrasexual

77 and intersexual information components, plus a mixed term provoked by the

78 confounding effect of the marginal frequencies when the mating propensity effects are

79 asymmetric. Interestingly, the three terms can be separately estimated from the observed

80 frequencies and so, the researcher can study how different models and parameters

81 translate into the different mate choice components. Also, it is proposed that this setting

82 provides the baseline for solving the mate choice null hypothesis problem, since the null

83 model emerges naturally from the idea of zero information. Thus, the correct null

84 should not rely on neutral preference or trait genes but on zero information.

85 The utility of this framework is shown by analyzing a real data example. I will show

86 how the view obtained from the unveiled relationships can be utilized to classify

87 different general models from its consequences which facilitates the multimodel

88 inference of the mate choice. However, a deeper study on the outcomes of different

89 forms of the mating preference functions is out of the scope of the present article and is

90 part of a different paper.

92 2. Model of Mate Choice

93 As mentioned above, the following model is as a particular specification of the

94 information theory interpretation for evolutionary models, proposed in (Frank, 2012b;

95 Frank, 2013). The general framework developed by this author fits perfectly for the

96 purpose of describing the occurrence of non-random mating and the flow of information

97 that it provokes. Remarkably, once the basic equation for the gain in information due to

98 non-random mating is formalized, the relationship between mate choice and its different 
99 evolutionary outcomes emerges naturally, providing a clear and useful picture of the

100 intrasexual and intersexual selection effects.

101

\subsection{General model}

103 Let consider a population with a number of $n_{1}$ females and $n_{2}$ males. For a given female

104 phenotype $X$ (e.g. shell color) with $K$ different classes having values $X_{1}, X_{2} \ldots X_{\mathrm{k}}$, the

105 frequency of the phenotype $X_{\mathrm{i}}$ in the female population is $p_{1 \mathrm{i}}=n_{1 X \mathrm{i}} / n_{1}$, i.e. the number

106 of females with that phenotypic value divided by the total number of females. Similarly,

107 for the male phenotype $Y$ (could be the same as $X$ ) with $K^{\prime}$ classes, the frequency of $Y_{\mathrm{j}}$ in

108 the male population is $p_{2 \mathrm{j}}=n_{2 Y \mathrm{j}} / n_{2}$.

109 In this way, by using the frequency of the phenotype for each sex, the expected mating

110 frequencies if mating is at random is

111

$$
q_{\mathrm{ij}}=p_{1 \mathrm{i}} \times p_{2 \mathrm{j}}
$$

112 Now, given a female phenotype $X_{\mathrm{i}}$ and a male phenotype $Y_{\mathrm{j}}$, let's define the mutual

113 mating propensity $m_{\mathrm{ij}}(x, y, e)$ as the number of matings of $X_{\mathrm{i}}$ with $Y_{\mathrm{j}}$ after their

114 encounter in the environment $e$. The normalized mating propensity is

$$
m_{\mathrm{ij}}(x, y, e) / M
$$

116 where $M=\sum_{i, j} q_{i j} m_{i j}(x, y, e)$.

117 Then, the observed mating frequencies in a given environment $e$ can be expressed as

$$
q_{i j}^{\prime}=q_{i j} \frac{m_{i j}(x, y, e)}{M}
$$


119 Therefore, the observed mating frequencies are the result of the functions $m_{\mathrm{ij}}(x, y, e)$

120 (hereafter noted as $m_{\mathrm{ij}}$ ), that can be any kind of composition of the preference of female

$121 X_{\mathrm{i}}$ for male $Y_{\mathrm{j}}$, and vice versa, in the environment $e$.

122 Note that random mating is a particular case of the model in (1) when the propensities

123 are equal for every mating pair. The mutual mating propensity functions can represent

124 empirical or analytical functions, as for example the Gaussian-like preference functions

125 (reviewed in Carvajal-Rodriguez and Rolán-Alvarez, 2014). Moreover, each $m_{\mathrm{ij}}$ can be

126 composed of female and male preferences, so mutual mate choice models (Bergstrom

127 and Real, 2000) are also available under this setting. The standardized $m_{\mathrm{ij}}$ values could

128 also be estimated a posteriori from the data. In this case they coincide with the pair total

129 index i.e. the ratio of the frequency of the observed types divided by the expected pair

130 types calculated from the total frequencies $\left(P T I_{\mathrm{ij}}=q_{\mathrm{ij}}^{\prime} / q_{\mathrm{ij}}\right.$, Rolán-Alvarez and Caballero,

131 2000) which becomes an observation of the mutual mating propensity from the mating

132 phenotypes (see below).

133 Once we have the mating frequencies as defined in (1), the change with respect to

134 random mating is

$$
\Delta q_{i j}=q_{i j}^{\prime}-q_{i j}=q_{i j}\left(\frac{m_{i j}}{M}-1\right)
$$

136 The mean population change for a combined phenotype $\mathrm{Z}=\mathrm{X} * \mathrm{Y}$ is

$$
\Delta Z=\sum_{i, j} \Delta q_{i j} \mathrm{Z}_{\mathrm{ij}}
$$

137 Because the relationship in (1) is defined by ratios is more natural to express the

138 quantities in the logarithmic scale and so we can express $m_{\mathrm{ij}}$ as 


$$
m_{i j}=M \frac{q_{i j}^{\prime}}{q_{i j}}
$$

140 which in the logarithmic scale becomes

$$
l_{i j}=\log \left(m_{i j}\right)=\log (M)+\log \left(\frac{q_{i j}^{\prime}}{q_{i j}}\right)
$$

142 Thus, if we take the logarithm of the propensity as the combined phenotype $Z$ and by 143 noting that $\Sigma \Delta q_{\mathrm{ij}}=0$ and that $\log (M)$ is constant through the summation, then we can

144 measure the mean population change $\Delta L$ in relative propensity as

$$
\Delta L=\sum_{i, j} \Delta q_{i j} l_{i j}=\sum_{i, j} \Delta q_{i j} \log \left(\frac{q_{i j}^{\prime}}{q_{i j}}\right)=J\left(q^{\prime}, q\right) \equiv J_{P T I}
$$

146 which is the Kullback-Leibler symmetrized divergence (noted as Jeffreys in Frank,

147 2012b), that measures the gain in information when the differential mating propensity

148 moves the population from mating frequencies $q$ to $q^{\prime}$ or vice versa. Note that if the

149 propensity is equal for every pair i.e. $M=m_{\mathrm{ij}} \forall i, j$ then $q^{\prime}=q$ so that $J=0$ which is the

150 minimum information value since $J$ cannot be negative.

151 Recall from equation (1), that each $m_{\mathrm{ij}} / M$ is the ratio of the frequency of the observed

152 types divided by the expected pair types from the total frequencies. This is, by

153 definition, the pair total index PTI (Rolán-Alvarez and Caballero, 2000) and so, the

$154 \operatorname{logarithmic}$ term in $\Delta L$ is the logarithm of the $P T I$ values. Therefore, $J\left(q^{\prime}, q\right)$ measures

155 the gain in information as captured by the PTI coefficients, confronting the hypothesis

156 of mate choice against random mating. Hereafter, we note this $J$ as $J_{\mathrm{PTI}}$.

157 Interestingly enough, the Jeffreys' divergence computed as $J_{\mathrm{PTI}}$ (by taking the natural

158 logarithm and multiplying (2) by the total number of matings) is well approximated by a 
159 chi-square for the null hypothesis of random mating with $K K^{\prime}-1$ degrees of freedom

160 (Evren and Tuna, 2012).

161 The information obtained from $J_{\mathrm{PTI}}$ has been computed using the different propensities

162 as classes for classifying the couples i.e. we considered $\log (m)$ as the phenotype $Z$.

163 When the classes are based upon other phenotypes rather than propensities, we are

164 conveying a specific meaning for the change in frequencies, say, the change in mating

165 frequencies due to differential mutual propensities is observed in terms of change in

166 shell color mating frequencies. Therefore, the phenotype can be viewed as other scale

167 on which we can measure this information (Frank, 2013). Of course, different kinds of

168 phenotypes can be more or less involved in mate choice and so, different scales are

169 more or less useful for measuring the mate choice information.

\subsection{Relative propensity and phenotypes}

172 When we observe any mating pair $(i, j)$, we need to identify the mating by a given

173 characteristic (e.g. shell color) since we cannot directly classify it by the value of the

174 propensity function $m_{\mathrm{ij}}$. In general, we ignore the specific form of the mutual mating

175 propensity function $m$ and so, we may assume that some phenotype matches it perfectly,

176 as we did above (each phenotypic pair was perfectly differentiated by specific $m_{\mathrm{ij}}$

177 mating propensity).

178 Thus, if $T$ is the trait that is the target of the choice, we call $J_{\mathrm{PTI}}$ to the change in the numbers of matings when these matings were classified by $T$. 
181 Therefore, we may think on different traits $Z$ that classify the mating pairs; $Z$ can be a

182 composition of female trait $X$, e.g. preference, and male target $Y$, or can be any kind of

183 different traits or alternatively the same trait in both sexes as size, age or color. In any

184 case, we measure the mean change in $Z$ caused by differences in $m$, as

$$
\Delta_{m} Z=\sum_{\alpha, \beta} \Delta q_{\alpha \beta} Z_{\alpha \beta}=\sum_{\alpha, \beta} q_{\alpha \beta}\left(m_{\alpha \beta}-M_{z}\right) \frac{Z_{\alpha \beta}}{M_{z}}=\frac{\operatorname{cov}\left(Z, m_{z}\right)}{M_{z}}
$$

186 Where cov is the population covariance in the sense of Price (1972) as highlighted in

187 Frank (2012a). The subscripts $\alpha, \beta$ emphasize that we are looking at pairs with observed

188 phenotypes that not necessarily are the phenotypes exactly connected with the choice.

189 Therefore, the propensities for the matings classified under these phenotypes can be

190 different to the propensities for the trait $T$, then we note $m_{\mathrm{z}}$ and $M_{\mathrm{Z}}$ to distinguish from

191 the propensities $(m)$ and mean propensity $(M)$ measured directly from the real choice

192 trait

193 Equation (3) is in fact, a form of the Price equation with a different mapping for the

194 populations involved. While the Price equation (Frank, 2012a; Price, 1972) describes

195 the change in phenotype between two connected ancestor and descendant populations;

196 in our equation (3), the mapping is between the random mating population and the one

197 obtained under a given mutual mating propensity scheme.

198 The variable $Z$ can be any desired trait including, as we assumed above, the logarithm of

199 the propensities. So, if we take $Z$ equal to the logarithm of $m$, then by substituting in (3)

200 we obtain the mean population change $\Delta L_{\mathrm{z}}$ as

$$
\Delta L_{\mathrm{z}}=\operatorname{cov}\left(m_{\mathrm{z}}, l_{\mathrm{z}}\right) / M_{\mathrm{z}}
$$

202 where the subscript $z$ indicates that the propensities are now indexed by the trait $Z$. 
203 Recalling the relationship in (2), we now define

$$
J Z_{\mathrm{PTI}} \equiv \Delta L_{\mathrm{z}}=\operatorname{cov}\left(m_{\mathrm{z}}, l_{\mathrm{z}}\right) / M_{\mathrm{z}}=\alpha_{\mathrm{Z}} J_{\mathrm{PTI}}
$$

where if $J_{\mathrm{PTI}}>0$

$$
\alpha_{\mathrm{z}}=\left(\beta\left(m_{\mathrm{z}}, l_{\mathrm{z}}\right) \times D_{\mathrm{z}}\right) /(\beta(m, l) \times D)
$$

with $l_{\mathrm{z}}=\log \left(m_{\mathrm{z}}\right), D_{\mathrm{z}}=V\left(m_{\mathrm{z}}\right) / M_{\mathrm{z}}, l=\log (m)$ and $D=V(m) / M$

or $\alpha_{\mathrm{z}}=1$ if $J_{\mathrm{PTI}}=0$.

209 Note that $D$ and $D_{\mathrm{z}}$ are the indexes of dispersion over the choice and $Z$ traits

210 respectively, so $\alpha_{z}$ is the quotient of the regressions multiplied by the index of

211 dispersion at each phenotypic scale.

212 From the point of view of the estimation with real data, if we cannot measure directly

213 the values of $m$ then we simply compute $J$ based on trait $Z$ and therefore we are really

214 computing $J Z_{\mathrm{PTI}}$.

215 In this case, note that the PTI coefficients are no longer the exact estimate of the mutual

216 mating propensities because the ratio of frequencies $q_{\alpha \beta}^{\prime} / q_{\alpha \beta}$ does not correspond to

$217 m_{\mathrm{ij}} / M$ but to $m_{\alpha \beta} / M_{\mathrm{z}}$ which is a proxy that would be more or less precise depending on

218 the importance of the measured phenotype over the mating choice. For example, if shell

219 size is driving mate choice, the measure of $J Z_{\mathrm{PTI}}(Z=$ shell size $)$ would correspond well

220 with $J_{\mathrm{PTI}}\left(J_{\mathrm{PTI}}>0 ; \alpha_{\mathrm{z}} \approx 1\right)$. However, if other phenotype as shell color has nothing to do

221 with mate choice (and is not correlated with shell size) then the measure of $J Z_{\mathrm{PTI}}(Z=$

222 shell color) would be zero $\left(J_{\mathrm{PTI}}>0 ; \alpha_{\mathrm{z}}=0\right)$. Further details about the distinction

223 between $J Z_{\mathrm{PTI}}$ and $J_{\mathrm{PTI}}$ are given in appendix $\mathrm{A}$. 
224 The mate choice mediated by the differences in mutual mating propensity would

225 produce a deviation from random mating. At the same time, this may cause two

226 different effects, namely, intrasexual selection and intersexual selection, hereafter noted

227 as sexual selection and sexual isolation, respectively.

\subsection{Sexual selection}

230 Sexual selection is defined as the observed change in gene or phenotype frequencies in

231 mated individuals with respect to total population frequencies (Rolán-Alvarez and

232 Caballero, 2000). This change can be studied using the frequencies within each sex, or

233 considering jointly both sexes, by using the pair sexual selection coefficient (PSS,

234 Rolán-Alvarez and Caballero, 2000). I will show that, when the PSS coefficients are

235 considered a priori as the marginal propensities for the mating types, the information

236 gained due to sexual selection is the sum of the information from each sex. When the

237 focus is on the phenotypes instead on the propensities, the partition continue to be true,

238 provided that the same phenotypic scale is applied when computing the PSS coefficients

239 and the intrasexual components.

240 From the general model, the population frequency of the female phenotype $X_{\mathrm{i}}$ is $p_{1 \mathrm{i}}$. The

241 observed frequency of $X_{\mathrm{i}}$ in mated individuals, $p^{\prime}{ }_{1 \mathrm{i}}$, is computed as the sum of the

242 mating frequencies involving a female $X_{\mathrm{i}}$

$$
p_{1 i}^{\prime}=\sum_{j}^{\text {males }} q_{i j}^{\prime}=p_{1 i} \sum_{j} p_{2 j} \frac{m_{i j}}{M}=p_{1 i} \frac{m_{f i}^{\prime}}{M}
$$

244 where $m_{\text {fi }}^{\prime}$ is the marginal mating propensity for the female type $i$. 
245 Similarly for males, the frequency of phenotype $Y_{\mathrm{j}}$ is $p_{2 \mathrm{j}}$ and the frequency for the male

246 type $j$ in mated individuals is

$$
p_{2 j}^{\prime}=p_{2 j} \frac{m^{\prime} m j}{M}
$$

248 where $m_{\text {mj }}^{\prime}$ is the marginal mating propensity for the male type $j$.

249 The mean change in information due to sexual selection within each sex is, in terms of

250 the female marginal propensity (female intrasexual selection)

$$
\Delta_{m} L_{x}=\sum_{i}^{f \text { emales }} \Delta p_{1 i} X_{i}=\sum_{i j} p_{i}\left(m_{f i}^{\prime}-M\right) \frac{X_{i}}{M}=J\left(p_{1}^{\prime}, p_{1}\right) \equiv J_{S 1}
$$

and, in terms of male marginal propensity (male intrasexual selection)

$$
\Delta_{m} L_{y}=\sum_{j}^{\text {males }} \Delta p_{2 j} Y_{j}=J\left(p_{2}^{\prime}, p_{2}\right) \equiv J_{S 2}
$$

254 The term $J_{S}$ has been obtained in a similar way as for the general case, i.e. by expressing 255 each marginal $m_{\mathrm{fi}}^{\prime}$ and $m_{\mathrm{mj}}^{\prime}$ in function of their respective ratio of frequencies multiplied 256 by the mean propensity $M$ and substituting the phenotype $X$ or $Y$, by the logarithm of the corresponding (female or male) marginal $m^{\prime}$.

258 The change to the scale of phenotypes produces

$$
\Delta_{m} \bar{X}=\alpha_{x} J_{S 1} \equiv J X_{S 1}
$$

with

$$
\alpha_{\mathrm{x}}=\left[\beta\left(m_{\mathrm{xf}}^{\prime}, l_{\mathrm{xf}}\right) \times D_{\mathrm{xf}}\right] /\left[\beta\left(m_{\mathrm{f}}^{\prime}, l_{\mathrm{f}}\right) \times D_{\mathrm{f}}\right] \text { or } 1 \text { if } J_{\mathrm{PS} 1}=0
$$

where $l_{\mathrm{xf}}=\log \left(m_{\mathrm{xf}}^{\prime}\right)_{s} D_{\mathrm{xf}}=V\left(m_{\mathrm{xf}}^{\prime}\right) / M_{\mathrm{z}}, l_{\mathrm{f}}=\log \left(m_{\mathrm{f}}^{\prime}\right)_{\mathrm{s}} D_{\mathrm{f}}=V\left(m_{\mathrm{f}}^{\prime}\right) / M$.

263 And

$$
\Delta_{m} \bar{Y}=\alpha_{y} J_{S 2} \equiv J Y_{S 2}
$$




$$
\alpha_{\mathrm{y}}=\left[\beta\left(m_{\mathrm{ym}}^{\prime}, l_{\mathrm{ym}}\right) \times D_{\mathrm{ym}}\right] /\left[\beta\left(m_{\mathrm{m}}^{\prime}, l_{\mathrm{m}}\right) \times D_{\mathrm{m}}\right] \text { or } 1 \text { if } J_{\mathrm{PS} 2}=0
$$

266 where $l_{\mathrm{ym}}=\log \left(m_{\mathrm{ym}}^{\prime}\right), D_{\mathrm{ym}}=V\left(m_{\mathrm{ym}}^{\prime}\right) / M_{\mathrm{z}}$.

267 Note that the subscripts $x$ (females) or $y$ (males) refer to the matings classified by

268 phenotype instead of the true choice trait, also note that the mean of both female and

269 male marginals is the same and equal to the mean propensity $\left(M_{\mathrm{Z}}\right.$ or $M$ depending on the 270 scale).

$271 J X_{\mathrm{S} 1}$ and $J Y_{\mathrm{S} 2}$ are the Jeffrey's divergence that expresses the gain of information due to

272 intrasexual selection measured on the combined phenotypic scale $Z$.

\subsection{Pair sexual selection}

274 In addition to the computation within each sex, we can compare the expected pair types

275 under random mating calculated in mated individuals, with the expected pair types from

276 total numbers $\left(P S S\right.$, see above). Thus, $P S S_{\mathrm{ij}}=\left(p^{\prime}{ }_{1 \mathrm{i}} p^{\prime}{ }_{2 \mathrm{j}}\right) /\left(p_{1 \mathrm{i}} p_{2 \mathrm{j}}\right)=m_{\mathrm{fi}}^{\prime} m_{\mathrm{mj}}^{\prime} / M^{2}$. The latter

277 term can be viewed as an a priori expression of the PSS coefficients. Again, the

278 difference between the observed and the expected distribution can be expressed as

$$
\Delta_{P S S} L=\sum_{i, j} \Delta\left(p_{1 i} p_{2 j}\right) \log \left(\frac{p_{1 i}^{\prime} p_{2 j}^{\prime}}{p_{1 i} p_{2 j}}\right)=J\left(p_{1}^{\prime} p_{2}^{\prime}, p_{1} p_{2}\right) \equiv J_{P S S},
$$

where $\Delta\left(p_{1 \mathrm{i}} p_{2 \mathrm{j}}\right)=p^{\prime}{ }_{1 \mathrm{i}} p^{\prime}{ }_{2 \mathrm{j}}-p_{1 \mathrm{i}} p_{2 \mathrm{j}}$

281 In the scale of phenotypes

$$
\Delta_{P S S} L_{\mathrm{z}}=\alpha_{\mathrm{psS}} J_{P S S} \equiv J Z_{P S S}
$$

with

$$
\alpha_{\mathrm{pss}}=\left(\beta\left(m_{\mathrm{z}}^{\prime}, l_{\mathrm{z}}^{\prime}\right) \times D_{\mathrm{z}}^{\prime}\right) /\left(\beta\left(m^{\prime}, l^{\prime}\right) \times D^{\prime}\right) \text { or } 1 \text { if } J_{\mathrm{PSS}}=0
$$


285 where $m_{\mathrm{z}}^{\prime}=m_{\mathrm{xf}}^{\prime} \times m_{\mathrm{ym}}^{\prime}, m^{\prime}=m_{\mathrm{f}}^{\prime} \times m_{\mathrm{m}}^{\prime}, D_{\mathrm{z}}^{\prime}=V\left(m_{\mathrm{z}}^{\prime}\right) / M_{\mathrm{z}}^{2}, l_{\mathrm{z}}^{\prime}=\log \left(m_{\mathrm{z}}^{\prime}\right), l^{\prime}=\log \left(m^{\prime}\right), D^{\prime}$

$286=V\left(m^{\prime}\right) / M^{2}$.

287 The change in the phenotype due to sexual selection is driven by the aprioristic version

288 of $P S S$, and is expressed in term of the information accumulated and rescaled from the

289 marginal propensities to $Z$.

290 The relationship between sexual selection measured within sex and the pair sexual

291 selection measured by PSS is (details in Appendix B)

292

$$
J_{\mathrm{PSS}}=J_{\mathrm{S} 1}+J_{\mathrm{S} 2}
$$

293 And in the scale of phenotypes

$$
J Z_{\mathrm{PSS}}=J X_{\mathrm{S} 1}+J Y_{\mathrm{S} 2}
$$

295

provided that the same phenotypic scale applies in the pair sexual selection statistic and in the intrasexual components (i.e. the criteria utilized for classifying the different

297 couples is the same).

298 The information captured in the PSS coefficients is the sum of the sexual selection

299 within each sex.

\subsection{Sexual isolation}

302 Sexual isolation is defined as the deviation from random mating in mated individuals

303 (Rolán-Alvarez and Caballero, 2000). The pair sexual isolation statistic (PSI) is the

304 number of observed pair types divided by the expected pair types from mates. In terms

305 of our model this is the ratio of frequencies 


$$
P S I_{\mathrm{ij}}=q_{\mathrm{ij}}^{\prime} /\left(p^{\prime}{ }_{1 \mathrm{i}} p_{2 \mathrm{j}}^{\prime}\right)=\left(m_{\mathrm{ij}} / M\right) /\left(m_{\mathrm{fi}}^{\prime} m_{\mathrm{mj}}^{\prime} / M^{2}\right) \equiv \delta_{\mathrm{ij}}
$$

307 The term $\delta$ refers to an aprioristic (depends on the $m$ 's from the model) definition of the

308 PSIs. The joint isolation index for PSI can be expressed as

$$
I_{P S I}=\frac{(k-1) \sum_{i} \delta_{i i}-\sum_{i j} \delta_{i j}}{(k-1) \sum_{i} \delta_{i i}+\sum_{i j} \delta_{i j}}
$$

310 where $k$ is the number of phenotypic classes involved in the classification of the matings

311 (Carvajal-Rodriguez and Rolan-Alvarez, 2006).

312 As with the previous pairwise statistics, we may obtain the equations of change between observed and expected pair types in terms of $J$.

$$
\Delta_{P S I} L=\sum_{i, j} \Delta\left(p_{1 i}^{\prime} p_{2 j}^{\prime}\right) \log \left(\frac{q_{i j}^{\prime}}{p_{1 i}^{\prime} p_{2 j}^{\prime}}\right)=J\left(q^{\prime}, p_{1}^{\prime} p_{2}^{\prime}\right) \equiv J_{P S I}
$$

315 where $\Delta\left(p^{\prime}{ }_{1 \mathrm{i}} p^{\prime}{ }_{2 \mathrm{j}}\right)=q_{\mathrm{ij}}^{\mathrm{ij}}-p^{\prime}{ }_{1 \mathrm{i}} p^{\prime}{ }_{2 \mathrm{j}}$.

316 In the scale of phenotypes

$$
\Delta_{P S I} L_{z}=\alpha_{\delta} J_{P S I} \equiv J Z_{P S I}
$$

318 The scaling factor $\alpha_{\delta}$ is not always easy to compute. Provided that there is no sexual

319 selection $\left(J_{\mathrm{PSS}}=0\right)$ then $\alpha_{\delta}=\alpha_{\mathrm{z}}$ and so $J Z_{\mathrm{PTI}}=J Z_{\mathrm{PSI}}=\alpha_{\mathrm{z}} J_{\mathrm{PSI}}$. Otherwise we need to

320 rescale the factor $E_{0}$ (see below) to finally get the transformation between $J Z_{\mathrm{PSI}}$ and $J_{\mathrm{PSI}}$

321 (see Appendix $C$ ).

322 The $J Z_{\mathrm{PSI}}$ index provides the correct metric to express the part of change in mating

323 information that translates into sexual isolation. Presenting the PSI's under this

324 formalism allow us to appreciate some facts that are not obvious from the a posteriori

325 definition of coefficients estimated from data. We must realize (see equation 5) that if the normalized propensity of each pair $\left(m_{\mathrm{ij}} / M\right)$ is the product of the normalized 
327 marginal types of each partner then $\delta=1$ and so, both, the values of $I_{\mathrm{PSI}}$ and $J_{\mathrm{PSI}}$ are

328 zero indicating no sexual isolation at all. Thus, in any model in which the mutual mating

329 propensity is multiplicative, the only possible outcome from mate choice is intrasexual

330 selection.

331 We can illustrate the multiplicative effect by means of a simple model based on a real

332 species scenario. The bird sage grouse (Centrocercus urophasianus) has elaborate

333 courtship rituals. In the spring season, males congregate in leks that are visited by the

334 females that actively choose one of the males for mating. The number of females

335 visiting a male seems to be related with the male long-range acoustic broadcasts

336 whereas the probability of mating once visited is related to the visual display (Gibson,

337 1996).

338 It has been suggested that both traits, acoustic broadcast and display rate, yield a

339 multiplicative preference for males with specific acoustic conditions and high display

340 rates (Gibson, 1996; Rosenthal, 2017).

341 Thus, we can define a model of the multiplicative effect of the aforementioned traits

342 (see details in Appendix D). Obviously, the real mating scenario is by far more

343 complex, but the example suffices to illustrate the point.

344 The females are the choosers and so our model assumes a single female phenotypic

345 class $(X)$ and two male traits with two phenotypic classes each, $B / b$ for acoustic

346 broadcast, and $D / d$ for display rate, where in both cases the upper case refers to the

347 higher value of the trait. We define a multiplicative preference effect for acoustic

348 broadcast and display rate, so that the female propensity for males $B D$ can be expressed

349 as the product of the female propensities for $B d$ and $d B$, i.e. $m_{\mathrm{XBD}}=m_{\mathrm{XBd}} \times m_{\mathrm{XbD}}$

350 (supplementary Table S1). 
351 Under this model, the mean propensity $M$ coincides with the female marginal, $m_{\mathrm{fX}}^{\prime}=M$.

352 The four male marginal propensities ( $\left.m_{\mathrm{mBd}}^{\prime}, m_{\mathrm{mBD}}^{\prime}, m_{\mathrm{mbD}}^{\prime}, m_{\mathrm{mbd}}^{\prime}\right)$ have the same values

353 as their corresponding mutual propensities $\left(m_{\mathrm{XBd}}=\alpha, m_{\mathrm{XBD}}=\alpha \beta, m_{\mathrm{XbD}}=\beta, m_{\mathrm{Xbd}}=1\right.$; see

354 Appendix D).

355 The model is multiplicative since each normalized mutual propensity is equal to the

356 product of the normalized marginals, e.g. $m_{\mathrm{XBd}} / M=\left(m_{\mathrm{fX}}^{\prime} / M\right) \times\left(m_{\mathrm{mBd}}^{\prime} / M\right)$.

357 By computing the aprioristic expressions in (5), we see that $\delta_{\mathrm{Bd}}=\delta_{\mathrm{BD}}=\delta_{\mathrm{bD}}=\delta_{\mathrm{bd}}=1$.

358 Thus, provided that the mating reflects the propensities, the result is that independently

359 of the phenotypes, the number of observed pair types would be equal to the expected

360 pair types from mates, which means that there is no sexual isolation.

361 On the other hand, the model predicts male sexual selection whenever $m_{\mathrm{XBd}}$ and/or $m_{\mathrm{XbD}}$ $362 \neq 1$.

\section{Relationship between Mate Choice, Sexual Selection and Sexual Isolation}

365 The information as captured by the PTI coefficients can be partitioned in terms of PSS and PSI. Recall the expression (2) for $J_{\mathrm{PTI}}$

$$
\Delta L=\sum_{i, j} \Delta q_{i j} \log \left(\frac{q_{i j}^{\prime}}{q_{i j}}\right)=J\left(q^{\prime}, q\right) \equiv J_{P T I}
$$

368 The term $\Delta q_{\mathrm{ij}}$ can be expressed as the sum of the frequency changes for sexual selection and isolation

$$
\Delta q_{\mathrm{ij}}=\Delta\left(p_{1 \mathrm{i}} p_{2 \mathrm{j}}\right)+\Delta\left(p^{\prime}{ }_{1 \mathrm{i}} p^{\prime}{ }_{2 \mathrm{j}}\right)
$$


371 The $\operatorname{logarithmic}$ term $\log \left(q_{\mathrm{ij}}^{\prime} / q_{\mathrm{ij}}\right)$ which we have also noted as $\log (P T I)$ is also

372 partitioned in the sexual selection and isolation components

$$
\log (P T I)=\log (P S S)+\log (P S I)
$$

Therefore

where $E_{0}=\Sigma_{\mathrm{ij}}\left(\Delta\left(p_{1 \mathrm{i}} p_{2 \mathrm{j}}\right) \log \left(P S I_{\mathrm{ij}}\right)+\Delta\left(p^{\prime}{ }_{1 \mathrm{i}} p^{\prime}{ }_{2 \mathrm{j}}\right) \log \left(P S S_{\mathrm{ij}}\right)\right)$. However, note that

$\left.\Delta\left(p^{\prime}{ }_{1 i} p^{\prime}{ }_{2 \mathrm{j}}\right) \log \left(P S S_{\mathrm{ij}}\right)\right)=0$ (see Appendix E) so finally

$$
J_{\mathrm{PTI}}=\Sigma_{\mathrm{ij}}\left(\left[\Delta\left(p_{1 \mathrm{i}} p_{2 \mathrm{j}}\right)+\Delta\left(p^{\prime}{ }_{1 \mathrm{i}} p^{\prime}{ }_{2 \mathrm{j}}\right)\right] \times\left[\log \left(P S S_{\mathrm{ij}}\right)+\log \left(P S I_{\mathrm{ij}}\right)\right]\right) \text { i.e. }
$$

$$
J_{\mathrm{PTI}}=J_{\mathrm{PSS}}+J_{\mathrm{PSI}}+E_{0}
$$
logarithm of the PSIs.

$$
\alpha_{\mathrm{E}}=\beta\left(m_{\mathrm{z}}^{\prime}, l_{\mathrm{zpsi}}\right) \times D_{\mathrm{z}}^{\prime} / \beta\left(m^{\prime}, l_{\mathrm{psi}}\right) \times D^{\prime} \text { or } 1 \text { if } E_{0}=0
$$

where $l_{\mathrm{zpsi}}=\log \left(P S I_{\mathrm{z}}\right), l_{\mathrm{psi}}=\log (P S I)$ and $m_{\mathrm{z}}^{\prime}, m^{\prime}, D_{\mathrm{z}}^{\prime}$ and $D^{\prime}$ are the same as defined for $\alpha_{\mathrm{pss}}$. The subscript in $P S I_{\mathrm{z}}$ indicates that this is the value obtained under trait $Z$ contrary to PSI which is obtained directly under the choice trait.

387 Then, $\alpha_{\mathrm{E}}$ permits to interchange between the scale of phenotypes and choice so, $Z E_{0}=$ $\alpha_{\mathrm{E}} E_{0}$.

389 Alternatively, we can also express $E_{0}$ (see Appendix E for details) as

$$
E_{0}=D_{\mathrm{KL}}^{\prime}\left(w, q^{\prime} \| p^{\prime}\right)
$$


391 which is a Kullback-Leibler-like divergence with weights $w_{\mathrm{ij}}=\left(P S S_{\mathrm{ij}}-1\right) / P T I_{\mathrm{ij}}$ in the

392 observations $q^{\prime}$. Note that contrary to the standard K-L divergence, $E_{0}$ can be negative 393 depending on the weights.

394 The total information is separated into the sexual selection $\left(J_{\mathrm{PSS}}\right)$ and isolation $\left(J_{\mathrm{PSI}}\right)$

395 components plus the mixed term $E_{0}$. Note that $E_{0}$ appears only when both sexual

396 selection and sexual isolation effects occur so that the above given covariance is not

397 null

398 If $E_{0}=0$ this means that $J_{\mathrm{PSS}}$ and/or $J_{\mathrm{PSI}}$ capture the complete information from mate

399 choice. When $E_{0}$ is positive it indicates that the information gathered from $J_{\mathrm{PSS}}$ and $J_{\mathrm{PSI}}$

400 separately is not the total information from mating choice. On the other side, when $E_{0}$ is

401 negative there is some inverse relationship between sexual selection and sexual isolation

402 information.

403 In the scale of phenotypes the partition still holds provided that the same phenotypic

404 classification is applied when computing the different measures

$$
J Z_{\mathrm{PTI}}=J Z_{\mathrm{PSS}}+J Z_{\mathrm{PSI}}+Z E_{0}
$$

where $Z E_{0}$ is the value of $E_{0}$ in the given phenotypic scale.

407 For any given logarithmic base, the amount of the total information, $J Z_{\mathrm{PTI}}$, depends on

408 the magnitude of the differences among the mutual mating propensity values linked to

409 the population phenotypes under study. The higher the differences encountered the

410 higher the value of $J Z_{\mathrm{PTI}}$. Without loss of generality, from herein we consider the

411 natural logarithm because this facilitates testing against the null hypothesis of no

412 information by means of the chi-square distribution. 
413 We have given formulae for the change in the phenotypic scale for every term in (6)

414 except $J Z_{\mathrm{PSI}}$. In this case, we have to predict the change in the scale by computing the

415 remaining factors and solving for $J Z_{\mathrm{PSI}}$ (Appendix C).

416 If, as expected, the observations used to compute the information statistics come from

417 the same sample, the sum in (6) is exact so it recovers the whole information gathered

418 from mate choice. On the contrary, if the computations has been performed using

419 different samples, it could be a remaining part of mate choice information that is non-

420 explained by the above statistics but that can be recovered by the error term

$$
E_{\mathrm{PTI}}=J Z_{\mathrm{PTI}}-\left(J Z_{\mathrm{PSS}}+J Z_{\mathrm{PSI}}+Z E_{0}\right)
$$

that reflects how much information may be lost due to differences in the measurement

of the involved phenotypes when computing the different information components from separate samples.

\section{Real Data Application}

The theoretical framework I have presented so far has been defined in a general way, for any number of male and female phenotypic classes, and for any kind of mutual mating propensities. The application of the $J$ statistics to a data sample of dimorphic traits (two classes), is immediate. For clarity, I will use the same example that appears in the

431 pairwise statistics (PTI, PSI and PSS) original article (Rolán-Alvarez and Caballero,

432 2000). The correspondence between the pairwise statistics notation used by (Rolán-

433 Alvarez and Caballero, 2000) and ours is as follows. The two phenotypic types are

434 noted as $A$ and $B$, the total number of observed matings is $t$ and the number of $A$ type 435 females (A' in Rolán-Alvarez and Caballero, 2000) becomes, under our notation, $p_{1 \mathrm{~A}} n_{1}$, 
436 and so $B^{\prime}$ is $p_{1 \mathrm{~B}} n_{1}$; the number of $A$ males becomes $p_{2 \mathrm{~A}} n_{2}$ and $B$ males are $p_{2 \mathrm{~B}} n_{2}$. The

437 observed absolute number for each pair $(i, j)$ would be $q_{\mathrm{ij}}^{\prime} t$ with $i, j \in\{A, B\}$ (see Table

438 1). The total number of expected mating pairs from population frequencies is $n_{1} n_{2}$

439 corresponding to the quantity $S$ in (Rolán-Alvarez and Caballero, 2000).

TABLE 1. The mating model for two phenotypic classes identified as types $A$ and

\section{$B$. The number of observed mating pairs is $t$.}

\section{Females}

$\begin{array}{ccccc} & & p_{1 \mathrm{~A}} \mathrm{n}_{1} & p_{1 \mathrm{~B}} n_{1} \\ \text { Males } & p_{2 \mathrm{~A}} n_{2} & q_{\mathrm{AA}}^{\prime} t & q_{\mathrm{BA}}^{\prime} t & p_{2 \mathrm{~A}}^{\prime} t \\ & p_{2 \mathrm{~B}} n_{2} & q_{\mathrm{AB}}^{\prime} t & q_{\mathrm{BB}}^{\prime} t & p_{2 \mathrm{~B}}^{\prime} t \\ & & p^{\prime}{ }_{1 \mathrm{~A}} t & p^{\prime}{ }_{1 \mathrm{~B}} t & \end{array}$

$p_{1 \mathrm{i}}$ : observed relative frequency of type $i \in\{A, B\}$ in population females $; n_{1}$ : number of females in the

population; $p_{2 \mathrm{i}}$ : observed relative frequency of type $i \in\{A, B\}$ in population males $; n_{2}$ : number of males in frequency of type $i$ in mating males; $q_{\mathrm{ij}}^{\prime}$ : observed relative frequency of mating pair $i, j$.

448 The data correspond to a multiple-choice experiment involving two different lines of

449 Drosophila melanogaster so called M-like and Z-like (Hollocher et al., 1997). Rolán-

450 Alvarez \& Caballero applied the pairwise statistics to this data and confirmed the

451 previous results from Hollocher et al indicating stronger sexual isolation than sexual

452 selection. They also suggested a fitness advantage of females versus males but they

453 were not able of distinguishing between female sexual selection and male preference for $M$ females. 
455 To perform the analysis, we expressed the observed data from that experiment in terms

456 of the information model as presented in Table 1. In doing so, and noting that the

457 observed number of mating pairs was $t=1704$, we obtained the necessary quantities in

458 terms of our model (Table 2).

459 The total mate choice information obtained in $J Z_{\mathrm{PTI}}$ is partitioned in $89 \%$ of sexual

460 isolation $\left(J Z_{\mathrm{PSI}} / J Z_{\mathrm{PTI}}=0.468 / 0.526=0.89 ; I_{\mathrm{PSI}}=0.63\right), 6 \%$ of sexual selection and

$4615 \%$ of mixed effects which explains the $100 \%$ of $J Z_{\mathrm{PTI}}$. The information coming from

462 sexual isolation is 14 times that from sexual selection, result that matches pretty well the

463 outcome in (Rolán-Alvarez and Caballero, 2000).

464 The value of $J Z_{\mathrm{PTI}}$ multiplied by the number of matings can be approximated by a chi-

465 square variable with 3 degrees of freedom under the expectation of $J Z_{\text {PTI }}=0$, the $p$ -

466 value obtained was below 0.00001 which indicates non-random mating. The test against

$467 J Z_{\mathrm{PSI}}=0$ with 1 degree of freedom, also had a $p$-value below 0.00001 . The test against

$468 J Z_{\mathrm{PSS}}=0$ was also below 0.0001 . However, testing separately the female and male

469 sexual selection cases (with one degree of freedom each) produced a $p$-value below

$470 \quad 0.0001$ for the female case but a $p$-value of 0.77 for males.

471 Thus, we detected significant sexual isolation and selection effects as previously

472 reported by (Rolán-Alvarez and Caballero, 2000). The sexual selection component is

473 caused by a significant intrasexual effect in females. The mixed term $E_{0}$ is positive thus

474 indicating that not all the information is recovered by the PSS and PSI coefficients. This

475 is due to the confounding effect which explains as far as the 5\% from the total

476 information. 
TABLE 2. Analysis using the mate choice information model (Table 1 and equations 7) on D. melanogaster mating data from (Hollocher et al., 1997). The

\begin{tabular}{|c|c|c|c|c|}
\hline & & Females Z & Females M & \\
\hline & & $0.5 \times 1440$ & $0.5 \times 1440$ & \\
\hline Males Z & $0.5 \times 1440$ & $0.3585 \times 1704$ & $0.145 \times 1704$ & $0.5035 \times 1704$ \\
\hline Males M & $0.5 \times 1440$ & $0.051 \times 1704$ & $0.4455 \times 1704$ & $0.4965 \times 1704$ \\
\hline & & $0.4095 \times 1704$ & $0.5905 \times 1704$ & \\
\hline$J Z_{\mathrm{PTI}}$ & 0.526 & & & \\
\hline$J Z_{\mathrm{PSI}}\left(I_{\mathrm{PSI}}\right)$ & $0.468(0.63)$ & & & \\
\hline$J X_{\mathrm{S} 1}+J Y_{\mathrm{S} 2}$ & $0.033+0.00005$ & & & \\
\hline$Z E_{0}$ & 0.024 & & & \\
\hline$E_{\mathrm{PTI}}$ & 0 & & & \\
\hline
\end{tabular}

481 Number of females in the population: $n_{1}=1440$; number of males in the population: $n_{2}=1440$; From

482 Table $1, p^{\prime}{ }_{11}$ : observed relative frequency of type $i$ in mating females; $p_{2 \mathrm{i}}{ }_{\mathrm{i}}$ : observed relative frequency of 483 type $i$ in mating males; $q_{\mathrm{ij}}^{\prime}$ : observed relative frequency of copulating pair $i, j$.

486 In the analysis performed above we used the information partition for testing if the

487 observations can be explained by random mating, in a similar way as we do when using

488 the $I_{\mathrm{PSI}}$ statistic for testing sexual isolation (Carvajal-Rodriguez and Rolan-Alvarez, 489 2006; Rolán-Alvarez and Caballero, 2000).

490 However, the proposed theoretical framework permits going further than just testing 491 random mating. We can rely on the described properties of mutual propensities under 
492 sexual selection and isolation, for defining different effects models. If we can define

493 models from which we can predict the effects, then we can try to fit and infer significant

494 parameters from the available data.

495 As an example, I have used the software InfoMating (Carvajal-Rodriguez, 2017) to

496 estimate the mutual mating propensity parameters associated to the data in Table 2 . The

497 software uses the $J$ information framework to a priori construct (before data) different

498 effects models, and then compare the fitting of random mating, sexual selection and

499 sexual isolation models to the data. There are models having sexual selection only in

500 females, only in males or in both. The models with sexual isolation will have or not

501 sexual selection depending on the frequencies (the conditions on marginal propensities

502 for sexual selection are frequency dependent). The most complex model is also

503 considered. Under this model the mutual mating propensities are estimated by the PTIs

504 that are indeed the maximum likelihood estimates.

505 I have considered BIC (Schwarz, 1978) and AIC (Akaike, 1973) selection criteria. Both

506 gave similar results. The best fit model was a two parameter model with sexual isolation

507 and female sexual selection effects. The model uses two parameters $a$ and $b$ to define

508 the four mating propensity values as $m_{\mathrm{ZZ}}=a, m_{\mathrm{ZM}}=1-b, m_{\mathrm{MZ}}=1, m_{\mathrm{MM}}=a+b$.

509 The obtained estimates under the BIC criterion were $a=2.47$ and $b=0.64$ which after

510 normalization, provide the mutual mating propensity estimates as they appear in Table

5113.

512 TABLE 3. Mutual-propensity estimates from multimodel inference.

Female $\mathbf{Z} \quad \mathbf{M}$

Male 


$$
\begin{aligned}
& \begin{array}{lll}
\mathbf{Z} & 1.42 & 0.58
\end{array} \\
& \begin{array}{lll}
\text { M } & 0.21 & 1.79
\end{array}
\end{aligned}
$$

514 The obtained estimates are almost identical to the corresponding PTI values but we have

515 only needed two parameters instead of three for defining the model. Therefore, the two

516 parameter model may provide some insight into the biology of the mating relationships.

517 The obtained estimates imply positive assortative mating because the homotype mutual

518 propensities (main diagonal in Table 3) are higher than the heterotype ones

519 (antidiagonal, $m_{\mathrm{ZM}}$ and $m_{\mathrm{MZ}}$ ). If we compare the mean homotype versus the mean

520 heterotype mating propensities, the difference is $a+b-1$. The value 1 is the value under

521 random mating so, the increase of homotype mating with respect to random mating is $a$

$522+b$

523 Moreover, the chosen model has no male sexual selection effect. This is clear when we

524 measure the mean effect of changing the male type in the matings. We see that the

525 effect is 0 i.e. $\left(m_{\mathrm{MM}}-m_{\mathrm{MZ}}+m_{\mathrm{ZM}}-m_{\mathrm{ZZ}}\right) / 2=0$. On the contrary, the mean effect of

526 changing female $\mathrm{Z}$ by $\mathrm{M}$ is $b$.

527 Thus, the deviation from random mating in the data from Table 2 is composed of a

528 sexual isolation effect captured by the parameter $a$ plus an effect $b$ of sexual selection

529 focused on the $\mathrm{M}$ females which may imply that this females are more receptive to

530 mating in general. 
533 The example we have considered involves the same trait in female and male. However,

534 there are several situations where the female preference is for a male display trait

535 (Pomiankowski and Iwasa, 1998). In this case, the female trait is the exerted preference

536 and the male trait is the target phenotype. In the preference-display context, the traits

537 involved are different between sexes so that the crosses cannot be classified in

538 homotypic versus heterotypic, which prevents the calculation of $I_{\mathrm{PSI}}$ and other similar

539 indices that are only applicable to mating models in which the female and male

540 phenotype is the same (similarity/dissimilarity models).

541 The mutual mating propensity framework can easily capture the preference-display

542 scenario to express the components of mate choice in terms of information.

543 In Table 4 we appreciate three examples of such preference-display models. There are

544 two types of females which have preference for males displaying phenotypic values $A$,

$545 B$ or $C$. The frequencies for the different phenotypes are equal. The mating propensities

546 have been defined with only one parameter and three possible values, namely $a, a / 2$ or

547 virtually $0(\varepsilon)$. In the first column the female preference generates a situation of

548 complete isolation; in the second column the resultant effect of the female preference is

549 of full intrasexual selection in males and the third column corresponds to a mixed

550 scenario were both sexual selection and isolation occur with a mixed effect of $-24 \%$

551 than indicates an strong overlap between both effects.

553 TABLE 4. Mating propensity models of female preference for male display traits.

554 Two types of females ' 0 ' or ' 1 ' might have different preferences for males 
preference) function $f_{1}$.

\begin{tabular}{lllll}
\hline $\mathrm{m}_{\text {female, male }}$ & Isolation & Sexual selection & Mixed & Generic \\
\hline$m_{0 \mathrm{~A}}$ & $a$ & $a$ & $a$ & $f_{1}(0, \mathrm{~A})$ \\
$m_{0 \mathrm{~B}}$ & $a / 2$ & $a / 2$ & $\varepsilon$ & $f_{1}(0, \mathrm{~B})$ \\
$m_{0 \mathrm{C}}$ & $\varepsilon$ & $\varepsilon$ & $\varepsilon$ & $f_{1}(0, \mathrm{C})$ \\
$m_{1 \mathrm{~A}}$ & $\varepsilon$ & $a$ & $\varepsilon$ & $f_{1}(1, \mathrm{~A})$ \\
$m_{1 \mathrm{~B}}$ & $a / 2$ & $a / 2$ & $\varepsilon$ & $f_{1}(1, \mathrm{~B})$ \\
$m_{1 \mathrm{C}}$ & $a$ & $\varepsilon$ & $a$ & $f_{1}(1, \mathrm{C})$ \\
$J Z_{\mathrm{PTI}}$ & 13 & 13 & 26 & \\
$J Z_{\mathrm{PSI}} / J Z_{\mathrm{PTI}}$ & $100 \%$ & $0 \%$ & $75 \%$ & \\
$\left(J X_{\mathrm{S} 1}+J Y_{\mathrm{S} 2}\right) / J Z_{\mathrm{PTI}}$ & $0 \%$ & $100 \%(0 \%+100 \%)$ & $49 \%(0 \%+100 \%)$ & \\
$Z E_{0} / J Z_{\mathrm{PTI}}$ & $0 \%$ & $0 \%$ & $-24 \%$ &
\end{tabular}

560 However, upon inspecting the propensities in the Table 4, the effects (isolation,

561 selection, and mixed) of the preference-display scenarios are not so intuitive, which

562 stresses the usefulness of the information partition. For example, the column "Mixed"

563 can be represented in a two-way table (Table 5).

565 Table 5. Mating preferences involved in the mixed model from Table 4. 


\section{Females}

0

1 a

$\varepsilon$ $\varepsilon$

$\varepsilon$ $\varepsilon$

a

566

567 The pattern in Table 5 is a clear case of isolation that splits females 0 and males A from

568 females 1 and males C. Recall that a mixed model implies isolation+ sexual selection.

569 The model is mixed because there is strong sexual selection against B males that

570 virtually do not mate. At this stage, we do not care if this is because A and C have more

571 vigour than B in the searching for mates, or because females in general do not like B

572 males. The result is male sexual selection (against B males), so the model is mixed

573 because the preferences in the model produce both sexual isolation and selection.

574 We can perform a similar exercise with the other models in Table 4 and see, for

575 example, that the isolation model provokes isolation because females 0 prefer A while

576 skip C, and vice versa, females 1 skip A and prefer C (both female phenotypes have the

577 same preference for B).

578 Under uniform frequencies in both sexes, this isolation model does not generate sexual

579 selection. The marginal propensity of females 0 and 1 is the same, $m_{\mathrm{f} 0}^{\prime}=m_{\mathrm{f} 1}^{\prime}=(a+a / 2$

$580+\varepsilon) / 3$ so there is no sexual selection in females. In males, the marginal propensity of A

581 and $\mathrm{C}$ is $(a+\varepsilon) / 2$ while is $a / 2$ for B males; they are equal except for the addition of the

582 factor $\varepsilon / 2$ which is virtually 0 and therefore there is no detectable effect of sexual

583 selection. 
586 The mate choice model defined in (1) is valid for phenotypes and genotypes, and it only

587 requires the abstract representation of any kind of relative mutual mating propensity.

588 The model in (1) is similar to the model for the mating pattern predicted from

589 encounter-mating (EM) scenarios when the availability of individuals is not affected by

590 the matings that have already occurred (equation 19 in Gimelfarb, 1988). The latter

591 happens in polygamous species, or even with monogamous, when only a small fraction

592 of individuals of both sexes successfully mate (i.e. the process of the encounter

593 corresponds to sampling with replacement).

594 On the contrary, when the species are monogamous and the population size is small, the

595 mating pattern will depend on the kind of pair formation process (Gimelfarb, 1988). In

596 the latter case, the information framework should still be valid but the equations must be

597 updated after each mating round. Therefore, the pair formation process without

598 replacement, would introduce some noise in the obtained mating patterns. The

599 application of the proposed methodology in such situations is left for future work.

600 At the same time, (1) is analogous to the Wright's selection equation for the change in

601 gene frequencies so, from the viewpoint of that analogy, the relative propensity would

602 play the role of fitness referred to each mating couple. By defining the relationship

603 between observed and expected mating frequencies as a function of relative mating

604 propensity, the choice is expressed as a potentiality which is also a key characteristic of 605 fitness (Wagner, 2010).

606 As with the fitness concept, the mate propensity faces two main aspects, namely the

607 measurement of differences between couples, and the intrinsic causes that provokes the 608 propensity values. By expressing the equation of change in terms of the choice

609 information and its components, this work focused in the first aspect. 
610 I have connected the cause of mating choice, which is modeled by the abstract concept

611 of mutual mating propensity, with the different possible outcomes. Notably, the

612 connection between mate choice and its consequences appears in terms of information.

613 The general equation $\left(J_{\mathrm{PTI}}\right)$ represents the information gained by mate choice with

614 respect to random mating. This general information is the sum of the information due to

615 sexual isolation and sexual selection, plus a mixed effect term that can be computed

616 separately from the others. The mixed term measures the adjustment of the partition

617 components with respect to the total mate choice information. In addition, the

618 information from sexual selection is the sum of the male and female intrasexual

619 selection information.

620 Although the model has been constructed assuming discrete phenotypes, it is possible to

621 estimate the Kullback-Leiblerg divergence for the continuous case (Pérez-Cruz, 2008)

622 in order to apply a similar mate choice information partition for quantitative traits.

623 The information framework also provides a baseline for defining adequate null

624 hypotheses for the distinct aspects of the mate choice problem. In fact, the information

625 terms are mean log-likelihood ratios, so we can apply them for contrasting the different

626 null hypothesis about random mating, sexual selection, and isolation.

627 Therefore, the statistical test defined as $n J$ (total number of matings $n$, multiplied by the

628 Jeffreys' divergence) is similar to a $G$-test. In fact, if we note $G$ for the $G$-test with $n q$

629 expected counts, and $G^{\prime}$ for the $G$-test with $n q^{\prime}$ expected counts, then $n J=\left(G+G^{\prime}\right) / 2$.

630 Indeed, it has been shown that the $G$-test $G$, is highly correlated (0.99) with the Jeffreys'

631 statistic (Evren and Tuna, 2012).

632 We can perform the test against random mating by considering a chi-square distribution

633 with $K K^{\prime}-1$ degrees of freedom (Evren and Tuna, 2012; Sokal and Rohlf, 1981), where 
$634 K \times K^{\prime}$ is the number of different mating categories. The intrasexual selection

635 components correspond to $K-1$ and $K^{\prime}-1$ degrees of freedom for $K$ female and $K^{\prime}$ male

636 traits respectively. In addition, the sexual isolation component corresponds to $(K-1)\left(K^{\prime}\right.$ -

637 1) degrees of freedom.

638 Of course, we may also use randomization tests if we prefer to rely on the empirical

639 distribution approach.

640 Therefore, if we want to contrast mate choice for a given trait $Z$, we test deviations from

641 zero information in $J Z_{\text {PTI }}$ and its components. However, if we want to contrast mate

642 choice in general, we must test deviations from zero information in $J_{\text {PTI }}$ which should be

643 the same that testing a flat preference function across all trait values (Edward, 2015).

644 In addition to contrasting the null hypothesis of random mating, we may take advantage

645 of the informational partition of mate choice effects to develop different kind of general

646 models defined by their effects. This is possible because the developed relationships

647 expose and clarify useful general properties, such as the requirement of non-

648 multiplicative mutual propensity functions for obtaining sexual isolation effects and the

649 connection of the marginal propensities at each sex with sexual selection.

650 As an example of the possible insight that can be gained relying in the informational

651 framework, I reanalyzed the well-known example of D. melanogaster mating data from

652 (Hollocher et al., 1997) and besides confirming previous results on the components of

653 sexual isolation and selection effects, I have been able to fit a simple two-parameter

654 model that explains the data by means of a component of sexual isolation plus a sexual

655 selection component favoring the mating of the M-type females.

656 In addition to the similarity models in which the same phenotype is involved in both

657 sexes, the preference-display models are also easily interpreted in terms of information 
658 and we have been able of inspecting models of full isolation, full intrasexual selection,

659 and mixed effect models.

660 We have also seen an example with multiplicative mutual propensity by means of a

661 simple preference-display model based on the bird sage grouse (Centrocercus

662 urophasianus) in which the traits 'acoustic broadcast' and 'display rate', act

663 multiplicatively over the preference.

664 To conclude, it is worth mentioning that the concept of mate choice is important in the

665 evolutionary theory and other disciplines. It has been approached from a diversity of

666 fields and inference methodologies, which has provoked that the terminology has not

667 always been very precise. This may have contributed to some confusion in terms of

668 causes and effects jointly with plenty discussion (Ah-King and Gowaty, 2016; Edward,

669 2015; Janicke et al., 2016; Roughgarden et al., 2015).

670 Here, I have shown that the mean change in the mating phenotypes can be expressed as

671 the information gained due to mate choice. Overall, the obtained results lead to the

672 suggestion that the information interpretation of mate choice is an interesting avenue

673 that may help to improve the study of the causes as well as the effects of this important

674 evolutionary phenomenon.

\section{Acknowledgement}

I would like to thank Carlos Canchaya and two anonymous reviewers for their valuable comments on the manuscript. This work was supported by Xunta de Galicia (Grupo de Referencia Competitiva, ED431C2016-037), Ministerio de Economía y Competitividad (BFU2013-44635-P and CGL2016-75482-P) and by Fondos FEDER ("Unha maneira de facer Europa"). 


\section{Appendix A. Relative propensity and phenotypes}

677 Let $T$ the trait that is the target of the choice while $Z$ is any other trait that can be more

678 or less related to the choice.

679 We call $J_{\mathrm{PTI}}$ to the change in the number of matings when these matings were classified

680 by $T$ (equation 2 in the main text). On the other hand, the change in the number of

681 matings when they were classified by $Z$ is $J Z_{\text {PTI. }}$.

682 If we cannot measure directly the values of $m$ (trait $T$ ) then we simply compute $J$ based

683 on trait $Z$ and therefore we are really computing

$$
J Z_{\mathrm{PTI}}=\alpha_{\mathrm{Z}} J_{\mathrm{PTI}}
$$

685 The scaling factor $\alpha_{z}$ is

$\alpha_{\mathrm{z}}=\left(\beta\left(m_{\mathrm{z}}, l_{\mathrm{z}}\right) \times D_{\mathrm{z}}\right) /(\beta(m, l) \times D)$ if $J_{\mathrm{PTI}}>0$; or 1 otherwise.

687 The values $D_{\mathrm{m}}=V(m) / M$ and $D_{\mathrm{z}}=V\left(m_{\mathrm{z}}\right) / M_{\mathrm{z}}$ are the indexes of dispersion over $T$ and $Z$

688 respectively; the subscript $z$ indicates that the matings were classified by phenotype $\mathrm{Z}$

689 instead of by $T$ (see Appendix $\mathrm{C}$ for more details in the scaling formulae).

690 The distinction between $J Z_{\mathrm{PTI}}$ and $J_{\mathrm{PTI}}$ matters because when the information produced

691 by mate choice is computed as $J Z_{\mathrm{PTI}}$, a value of zero could means that $\left.i\right) \operatorname{cov}\left(m_{\mathrm{z}}, l_{\mathrm{z}}\right)=0$

692 so $\alpha_{z}=0$ i.e. the trait $Z$ do not covariate with the differential propensities (the mating is

693 random with regard to $Z$ ) or, alternatively $i$ i) $J_{\mathrm{PTI}}=0$ meaning that there is no

694 differential mating propensity at all, i.e. the mating is random independently of the trait

695 we focused on. 
696 Let's see an example of the first situation i.e. there is mate choice but the trait $Z$ is not

697 involved in the mate choice process. Thus, assume that some unknown trait $X$ that is

698 involved in an assortative mating process exists. There are two phenotypic classes '1'

699 and ' 2 ' so that $m_{11}=2, m_{12}=1, m_{12}=1, m_{22}=2$; the phenotype frequencies are uniform

700 in males and females, $p=0.5$, and mean propensity $M=1.5$. This results in $J_{\mathrm{PTI}}=J_{\mathrm{PSI}}=$

7010.1155.

702 However, when counting the matings, we evaluated a phenotype $Z$ with classes $A / B$ that

703 are independent of the mating choice process. If the trait responsible of the mate choice

704 is uniformly distributed over the phenotypes $A / B$ (i.e. half of $A$ individuals have trait

705 value ' 1 ' and the other half have value ' 2 ' and the same is true for $B$ individuals) then the

706 expected preference for the phenotype pairs are

$m_{\mathrm{AA}}=m_{\mathrm{AB}}=m_{\mathrm{BA}}=m_{\mathrm{BB}}=\left(m_{11}+m_{12}+m_{21}+m_{22}\right) / 4=1.5=M=M_{\mathrm{z}}$

708 Thus it is clear that the normalized preferences are 1 and $J Z_{\mathrm{PTI}}=0$.

709 Consider now a different case in which $J Z_{\mathrm{PTI}} \neq 0$, this means that the non-random

710 mating is correlated to some extent with the trait $Z$. For example, consider the same

711 mate choice scenario as above with $J_{\mathrm{PTI}}=J_{\mathrm{PSI}}=0.1155$ but now the phenotype under

712 study is partially linked to the mate choice so $m_{\mathrm{AA}}=1.7, m_{\mathrm{AB}}=1.2, m_{\mathrm{BA}}=1.2, m_{\mathrm{BB}}=$

$713 \quad 1.7$.

714 Recall that the frequencies are uniform. If we compute directly the information index

715 over the phenotypes $Z$, we get $J Z_{\mathrm{PTI}}=0.03$. The mean propensity for these phenotypes

716 is $M_{\mathrm{z}}=1.45$ and $\operatorname{cov}\left(m_{\mathrm{z}}, l_{\mathrm{z}}\right)=0.0435$. However, $M=1.5$ and $\operatorname{cov}(m, l)=0.1733$ for the

717 real mate choice trait $(T)$. The scaling is $\alpha_{\mathrm{z}}=\left[\operatorname{cov}\left(m_{\mathrm{z}}, l_{\mathrm{z}}\right) / M_{\mathrm{z}}\right] /[\operatorname{cov}(m, l) / M]=0.2597$

718 so $J_{\mathrm{PTI}}=J Z_{\mathrm{PTI}} / \alpha_{\mathrm{Z}}=0.03 / 0.2597=0.1155$, as expected. 
719 If we have an estimate or a computable proxy for the propensity function $m$, as for

720 example, a measure of distance between female and male traits $|\mathrm{D}|$, or a model with

721 Gaussian functions (Carvajal-Rodriguez and Rolán-Alvarez, 2014), then $J Z_{\mathrm{PTI}}$ and $J_{\mathrm{PTI}}$

722 can be estimated separately. We obtain $J_{\mathrm{PTI}}$ by means of $J\left(q^{\prime}, q\right)$ using the estimated

723 mating propensities to classify the frequencies, and we still can use the phenotypes $Z$ to

724 compute $J Z_{\mathrm{PTI}}$. The relationship between both measures may give an idea about the

725 linkage between the phenotypes $Z$ and the mate choice.

726 Suppose that the estimate of $J_{\mathrm{PTI}}$ is different from zero while $J Z_{\mathrm{PTI}}=0$, then mate choice

727 do exist but it is not linked with the phenotype $Z$. An interested researcher could

728 compare different traits looking for the ones having the best scaling for the information

$729 J_{\mathrm{PTI}}$, i.e. the one that is more involved in the mate choice. It seems that if we are able of

730 having good proxies for mating propensity, this could pave the way for testing the

731 impact of different traits on mate choice.

732 Additionally, we still can compute directly $\Delta Z=Z^{\prime}-Z$, i.e. the difference in phenotype

733 frequencies between observed and expected by random mating. Therefore, we have two

734 values, $\Delta_{\mathrm{m}} Z$ and $\Delta Z$, for the change in $Z$, the discrepancy between them gives an

735 estimate of the change in $Z$ caused by other factors than mating propensity (e.g.

736 predators) so $e_{\mathrm{Z}}=\Delta Z-\Delta_{\mathrm{m}} Z$.

737 Thus the total change in mean $Z$ is

$$
\Delta Z=\alpha_{z} J_{P T I}+e_{Z}
$$

\section{Appendix B}


739 The relationship between sexual selection measured within sex and the pair sexual

740 selection measured by $P S S$ is

741

$$
J_{\mathrm{PSS}}=J_{\mathrm{S} 1}+J_{\mathrm{S} 2}
$$

742 To see this, recall that $J_{P S S}$ is the sum of products $\Delta\left(p_{1 \mathrm{i}} p_{2 \mathrm{j}}\right) \times \log \left(\left(p_{1 \mathrm{i}}^{\prime} p_{2 \mathrm{j}}^{\prime}\right) /\left(p_{1 \mathrm{i}} p_{2 \mathrm{j}}\right)\right)$.

743 Then note that

$$
\log \left(\left(p^{\prime}{ }_{1 \mathrm{i}} p_{2 \mathrm{j}}^{\prime}\right) /\left(p_{1 \mathrm{i}} p_{2 \mathrm{j}}\right)\right)=\log \left(p^{\prime}{ }_{1 \mathrm{i}} / p_{1 \mathrm{i}}\right)+\log \left(p^{\prime}{ }_{2 \mathrm{j}} / p_{2 \mathrm{j}}\right)
$$

and that

$$
\Sigma_{\mathrm{ij}} \Delta\left(p_{1 \mathrm{i}} p_{2 \mathrm{j}}\right)=\Sigma_{\mathrm{ij}}\left(p^{\prime}{ }_{1 \mathrm{i}} p^{\prime}{ }_{2 \mathrm{j}}-p_{1 \mathrm{i}} p_{2 \mathrm{j}}\right)=\Sigma_{\mathrm{i}} p^{\prime}{ }_{1 \mathrm{i}} \Sigma_{\mathrm{j}} p^{\prime}{ }_{2 \mathrm{j}}-\Sigma_{\mathrm{i}} p_{1 \mathrm{i}} \Sigma_{\mathrm{j}} p_{2 \mathrm{j}}=0
$$

747 because each summation is 1 . Then, after some algebraic rearrangement we obtain

$$
\begin{aligned}
& J_{P S S}=\Sigma_{\mathrm{ij}} \Delta\left(p_{1 \mathrm{i}} p_{2 \mathrm{j}}\right) \log \left(\left(p^{\prime}{ }_{1 \mathrm{i}} p^{\prime}{ }_{2 \mathrm{j}}\right) /\left(p_{1 \mathrm{i}} p_{2 \mathrm{j}}\right)\right)=\Sigma_{\mathrm{i}} p^{\prime}{ }_{1 \mathrm{i}} \log \left(p^{\prime}{ }_{1 \mathrm{i}} / p_{1 \mathrm{i}}\right)-\Sigma_{\mathrm{i}} p_{1 \mathrm{i}} \log \left(p^{\prime}{ }_{1 \mathrm{i}} / p_{1 \mathrm{i}}\right)+ \\
& \Sigma_{\mathrm{j}} p^{\prime}{ }_{2 \mathrm{j}} \log \left(p^{\prime}{ }_{2 \mathrm{j}} / p_{2 \mathrm{j}}\right)-\Sigma_{\mathrm{j}} p_{2 \mathrm{j}} \log \left(p^{\prime}{ }_{2 \mathrm{j}} / p_{2 \mathrm{j}}\right)=J_{S 1}+J_{S 2} .
\end{aligned}
$$

\section{Appendix C. Scaling factors}

We can compute the scaling factors that translate the information between different phenotypic scales.

752 We have used the notation $J_{\mathrm{PTI}}, J_{\mathrm{PSS}}, J_{\mathrm{PSI}}$ and $E_{0}$ for indicating the information when measured from phenotypes that are the choice targets i.e. the phenotypes that the mates

754 care about in choosing each other. On the other hand, we note $J Z_{\mathrm{PTI}}, J Z_{\mathrm{PSS}}, J Z_{\mathrm{PSI}}$ and $Z E_{0}$ when the phenotypes may or may not be related with the choice.

756 Therefore, it is interesting to shown how the information changes between one measures or others. So that 
$J Z_{\mathrm{PTI}}=\alpha_{\mathrm{Z}} J_{\mathrm{PTI}} ; J Z_{\mathrm{PSS}}=\alpha_{\mathrm{pss}} J_{\mathrm{PSS}} ; J X_{\mathrm{S} 1}=\alpha_{\mathrm{x}} J_{\mathrm{S} 1} ; J Y_{\mathrm{S} 2}=\alpha_{\mathrm{y}} J_{\mathrm{S} 2} ; Z E_{0}=\alpha_{\mathrm{E}} E_{0}$ and $J Z_{\mathrm{PSI}}=\alpha_{\mathrm{z}} J_{\mathrm{PTI}}-$

$$
\left(\alpha_{\mathrm{pss}} J_{\mathrm{PSS}}+\alpha_{\mathrm{E}} E_{0}\right)
$$

760 The scalings are as follows.

761

$\alpha_{\mathrm{z}}=\left[\operatorname{cov}\left(m_{\mathrm{z}}, l_{\mathrm{z}}\right) / M_{\mathrm{z}}\right] /[\operatorname{cov}(m, l) / M]$ with $l_{\mathrm{z}}=\log \left(m_{\mathrm{z}}\right)$ and $l=\log (m)$ if $J_{\mathrm{PTI}}>0$

or $\alpha_{\mathrm{z}}=1$ otherwise $\left(J_{\mathrm{PTI}}=0\right)$.

763 However, we can also express $\operatorname{cov}\left(m_{\mathrm{z}}, l_{\mathrm{z}}\right) / M_{\mathrm{z}}$ as $\beta\left(m_{\mathrm{z}}, l_{\mathrm{z}}\right) \times D_{\mathrm{z}}$ which is the regression of

764 the propensity under the trait $\mathrm{Z}$ over its logarithm multiplied by the index of dispersion.

765 Then if $D_{\mathrm{m}}=V(m) / M$ and $D_{\mathrm{z}}=V\left(m_{\mathrm{z}}\right) / M_{\mathrm{z}}$ are the indexes of dispersion over the choice

766 and $Z$ traits respectively, we obtain

$$
\left(\operatorname{cov}\left(m_{\mathrm{z}}, l_{\mathrm{z}}\right) / M_{\mathrm{z}}\right) /(\operatorname{cov}(m, l) / M)=\left(\beta\left(m_{\mathrm{z}}, l_{\mathrm{z}}\right) \times D_{\mathrm{z}}\right) /(\beta(m, l) \times D)
$$

768 Then if if $J_{\mathrm{PTI}}>0$ define

$$
\alpha_{\mathrm{z}}=\left(\beta\left(m_{\mathrm{z}}, l_{\mathrm{z}}\right) \times D_{\mathrm{z}}\right) /(\beta(m, l) \times D)
$$

770

where $l_{\mathrm{z}}=\log \left(m_{\mathrm{z}}\right)$ and $l=\log (m)$

771

or $\alpha_{\mathrm{z}}=1$ if $J_{\mathrm{PTI}}=0$

772 Similarly,

$\alpha_{\mathrm{x}}=\left[\operatorname{cov}\left(m_{\mathrm{zf}}^{\prime}, l_{\mathrm{zf}}\right) / M_{\mathrm{z}}\right] /\left[\operatorname{cov}\left(m_{\mathrm{f}}^{\prime}, l_{\mathrm{f}}\right) / M\right]=\left(\beta\left(m_{\mathrm{zf}}^{\prime}, l_{\mathrm{zf}}\right) \times D_{\mathrm{zf}}\right) /\left(\beta\left(m_{\mathrm{f}}^{\prime}, l\right) \times D_{\mathrm{f}}\right)$

or 1 if $J_{\mathrm{PS} 1}=0$

where $l_{\mathrm{zf}}=\log \left(m_{\mathrm{zf}}^{\prime}\right)_{s} D_{\mathrm{zf}}=V\left(m_{\mathrm{zf}}^{\prime}\right) / M_{\mathrm{z}}, l_{\mathrm{f}}=\log \left(m_{\mathrm{f}}^{\prime}\right)_{s} D_{\mathrm{f}}=V\left(m_{\mathrm{f}}^{\prime}\right) / M$ 
778 where $l_{\mathrm{zm}}=\log \left(m_{\mathrm{zm}}^{\prime}\right), D_{\mathrm{zm}}=V\left(m_{\mathrm{zm}}^{\prime}\right) / M_{\mathrm{z}}$.

$\alpha_{\mathrm{pss}}=\left[\operatorname{cov}\left(m_{\mathrm{zf}}^{\prime} m_{\mathrm{zm}}^{\prime}, l_{\mathrm{z}}^{\prime}\right) / M_{\mathrm{z}}^{2}\right] /\left[\operatorname{cov}\left(m_{\mathrm{f}}^{\prime} m_{\mathrm{m}}^{\prime}, l^{\prime}\right) / M^{2}\right]=\left(\beta\left(m_{\mathrm{z}}^{\prime}, l_{\mathrm{z}}^{\prime}\right) \times D_{\mathrm{z}}^{\prime}\right) /\left(\beta\left(m^{\prime}, l^{\prime}\right) \times D^{\prime}\right)$

780 or 1 if $J_{\mathrm{PSS}}=0$

781 where $m_{\mathrm{z}}^{\prime}=m_{\mathrm{zf}}^{\prime} \times m_{\mathrm{zm}}^{\prime}, m^{\prime}=m_{\mathrm{f}}^{\prime} m_{\mathrm{m}}^{\prime}, D_{\mathrm{z}}^{\prime}=V\left(m_{\mathrm{z}}^{\prime}\right) / M_{\mathrm{z}}^{2}, l_{\mathrm{z}}^{\prime}=\log \left(m_{\mathrm{z}}^{\prime}\right), l^{\prime}=\log \left(m^{\prime}\right), D^{\prime}=$

$782 V\left(m^{\prime}\right) / M^{2}$.

783

$\alpha_{\mathrm{E}}=\left[\operatorname{cov}\left(m_{\mathrm{zf}}^{\prime} m_{\mathrm{zm}}^{\prime}, \log \left(P S I_{\mathrm{z}}\right)\right) / M_{\mathrm{z}}{ }^{2}\right] /\left[\operatorname{cov}\left(m_{\mathrm{f}}^{\prime} m_{\mathrm{m}}^{\prime}, \log (P S I)\right) / M^{2}\right]=\left(\beta\left(m_{\mathrm{z}}^{\prime}, l_{\mathrm{zpsi}}\right) \times D_{\mathrm{z}}^{\prime}\right) /$

$784 \quad\left(\beta\left(m^{\prime}, l_{\mathrm{psi}}\right) \times D^{\prime}\right)$ or 1 if $E_{0}=0$

785 where $l_{\mathrm{zpsi}}=\log \left(P S I_{\mathrm{z}}\right), l_{\mathrm{psi}}=\log (P S I)$.

786 Finally

787 if $J_{\mathrm{PSI}}>0$

$788 \alpha_{\delta}=\alpha_{\mathrm{z}}$ when $J_{\mathrm{PSS}}=0$ or in general

$\alpha_{\delta}=\frac{\alpha_{T} J Z_{P S I}}{\alpha_{p S S} \alpha_{E} J Z_{P T I}-\alpha_{Z}\left(\alpha_{E} J Z_{P S S}+\alpha_{p S S} Z E_{0}\right)}$

789 with $\alpha_{\mathrm{T}}=\alpha_{\mathrm{z}} \times \alpha_{\mathrm{pss}} \times \alpha_{\mathrm{E}}$; so that $J Z_{\mathrm{PSI}}=\alpha_{\delta} J_{\mathrm{PSI}}$.

\section{Appendix D. Multiplicative preference example}

790 Consider two male traits, $B / b$ for acoustic broadcast and $D / d$ for display rate, where in

791 both cases the upper case refers to the higher value of the trait; consider also one female

792 trait $X$ with a single phenotypic class. The females are the choosers and so we may

793 define multiplicative preferences as appear in Table S1. 
Table S1. Mating preferences for acoustic broadcast and display rate.

Males Bd

\section{Females}

$X$

$\alpha$
BD

$\alpha \beta$
bD

bd

796

797 The model assumes a single female phenotypic class and so the frequency is 1 . Let the

798 frequencies of the different male classes be $p_{\mathrm{Bd}}, p_{\mathrm{BD}}, p_{\mathrm{bD}}$ and $p_{\mathrm{bd}}$. Then the mean

799 preference is $M=\alpha p_{\mathrm{Bd}}+\alpha \beta p_{\mathrm{BD}}+\beta p_{\mathrm{bD}}+p_{\mathrm{bd}}$. There is only one female marginal

800 preference, that in fact coincides with the mean preference, $m_{\mathrm{fX}}^{\prime}=M$. There are four

801 male marginal preferences $\left(m_{\mathrm{mBd}}^{\prime}, m_{\mathrm{mBD}}^{\prime}, m_{\mathrm{mbD}}^{\prime}, m_{\mathrm{mbd}}^{\prime}\right)$ that match the corresponding

802 male propensity with the single female phenotype i.e. $m_{\mathrm{mBd}}^{\prime}=\alpha, m_{\mathrm{mBD}}^{\prime}=\alpha \beta, m_{\mathrm{mbD}}^{\prime}=\beta$

803 and $m_{\text {mbd }}^{\prime}=1$.

804 In (5) we have given an aprioristic expression $(\delta)$ of the pair sexual isolation (PSI)

805 coefficients in terms of the preferences so that $\delta_{\mathrm{ij}}=\left(m_{\mathrm{ij}} / M\right) /\left(m_{\mathrm{fi}}^{\prime} m_{\mathrm{mj}}^{\prime} / M^{2}\right)$. Therefore,

$806 \delta_{\mathrm{Bd}}=\left(m_{\mathrm{Bd}} / M\right) /\left(m_{\mathrm{fX}}^{\prime} m_{\mathrm{mBd}}^{\prime} / M^{2}\right)=(\alpha / M) /\left(M \alpha / M^{2}\right)=1$ and similarly for the other

807 phenotypes so finally $\delta_{\mathrm{Bd}}=\delta_{\mathrm{BD}}=\delta_{\mathrm{bD}}=\delta_{\mathrm{bd}}=1$, which implies no sexual isolation.

On the other hand, the model predicts male sexual selection whenever $\alpha$ and/or $\beta \neq 1$.

\section{Appendix E}

808

Proposition 1

809

$$
\Sigma_{\mathrm{ij}} \Delta\left(p^{\prime}{ }_{1 \mathrm{i}} p^{\prime}{ }_{2 \mathrm{j}}\right) \log \left(P S S_{\mathrm{ij}}\right)=0
$$

810 then 


$$
E_{0}=\Sigma_{\mathrm{ij}}\left(\Delta\left(p_{1 \mathrm{i}} p_{2 \mathrm{j}}\right) \log \left(P S I_{\mathrm{ij}}\right)+\Delta\left(p^{\prime}{ }_{1 \mathrm{i}} p^{\prime}{ }_{2 \mathrm{j}}\right) \log \left(P S S_{\mathrm{ij}}\right)\right)=\Sigma_{\mathrm{ij}} \Delta\left(p_{1 \mathrm{i}} p_{2 \mathrm{j}}\right) \log \left(P S I_{\mathrm{ij}}\right)
$$

812 First, recall that

$$
\Sigma_{\mathrm{ij}} \Delta\left(p^{\prime}{ }_{1 \mathrm{i}} p^{\prime}{ }_{2 \mathrm{j}}\right) \log \left(P S S_{\mathrm{ij}}\right)=\Sigma_{\mathrm{ij}}\left(q_{\mathrm{ij}}^{\prime}-p^{\prime}{ }_{1 \mathrm{i}} p^{\prime}{ }_{2 \mathrm{j}}\right) \log \left(P S S_{\mathrm{ij}}\right)
$$

814 and also that by definition of PSS

$$
\log \left(P S S_{\mathrm{ij}}\right)=\log \left(\left(p^{\prime}{ }_{1 \mathrm{i}} p^{\prime}{ }_{2 \mathrm{j}}\right) /\left(p_{1 \mathrm{i}} p_{2 \mathrm{j}}\right)\right)
$$

816 that can be expressed as

$$
\log \left(P S S_{\mathrm{ij}}\right)=\log \left(p^{\prime}{ }_{1 \mathrm{i}} / p_{1 \mathrm{i}}\right)+\log \left(p^{\prime}{ }_{2 \mathrm{j}} / p_{2 \mathrm{j}}\right)
$$

818 then by simple substitution and rearranging the terms

$$
\Sigma_{\mathrm{ij}} \Delta\left(p^{\prime}{ }_{1 \mathrm{i}} p^{\prime}{ }_{2 \mathrm{j}}\right) \log \left(P S S_{\mathrm{ij}}\right)=
$$

$$
\begin{aligned}
& \Sigma_{\mathrm{ij}}\left(q_{\mathrm{ij}}^{\prime}-p^{\prime}{ }_{1 \mathrm{i}} p^{\prime}{ }_{2 \mathrm{j}}\right) \log \left(P S S_{\mathrm{ij}}\right)=\Sigma_{\mathrm{ij}} q_{\mathrm{ij}}^{\prime} \log \left(p^{\prime}{ }_{1 \mathrm{i}} / p_{1 \mathrm{i}}\right)+\Sigma_{\mathrm{ij}} q_{\mathrm{ij}}^{\prime} \log \left(p^{\prime}{ }_{2 \mathrm{j}} / p_{2 \mathrm{j}}\right)- \\
& \Sigma_{\mathrm{ij}}\left(p^{\prime}{ }_{1 \mathrm{i}} p^{\prime}{ }_{2 \mathrm{j}}\right) \log \left(p^{\prime}{ }_{1 \mathrm{i}} / p_{1 \mathrm{i}}\right)-\Sigma_{\mathrm{ij}}\left(p^{\prime}{ }_{1 \mathrm{i}} p^{\prime}{ }_{2 \mathrm{j}}\right) \log \left(p^{\prime}{ }_{2 \mathrm{i}} / p_{2 \mathrm{i}}\right)
\end{aligned}
$$

822 Now recall that the $i$ subscript refers to females and subscript $j$ to males, then the double 823 summatory is the sum through females and males, thus by reminding that $\Sigma_{\mathrm{j}} p_{2 \mathrm{j}}^{\prime}=\Sigma_{\mathrm{i}} p^{\prime}{ }_{1 \mathrm{i}}$

$824=1$ we note that

$$
\Sigma_{\mathrm{ij}}\left(p^{\prime}{ }_{1 \mathrm{i}} p^{\prime}{ }_{2 \mathrm{j}}\right) \log \left(p^{\prime}{ }_{1 \mathrm{i}} / p_{1 \mathrm{i}}\right)=\Sigma_{\mathrm{i}}\left(p^{\prime}{ }_{1 \mathrm{i}}\right) \log \left(p^{\prime}{ }_{1 \mathrm{i}} / p_{1 \mathrm{i}}\right) \Sigma_{\mathrm{j}} p^{\prime}{ }_{2 \mathrm{j}}=\Sigma_{\mathrm{i}} p^{\prime}{ }_{1 \mathrm{i}} \log \left(p^{\prime}{ }_{1 \mathrm{i}} / p_{1 \mathrm{i}}\right)
$$

826 and similarly

$$
\Sigma_{\mathrm{ij}}\left(p^{\prime}{ }_{1 \mathrm{i}} p^{\prime}{ }_{2 \mathrm{j}}\right) \log \left(p^{\prime}{ }_{2 \mathrm{i}} / p_{2 \mathrm{i}}\right)=\Sigma_{\mathrm{j}} p^{\prime}{ }_{2 \mathrm{j}} \log \left(p^{\prime}{ }_{2 \mathrm{i}} / p_{2 \mathrm{i}}\right)
$$

so we have

$$
\Sigma_{\mathrm{ij}} \Delta\left(p^{\prime}{ }_{1 \mathrm{i}} p^{\prime}{ }_{2 \mathrm{j}}\right) \log \left(P S S_{\mathrm{ij}}\right)=
$$


831 Now note that

832

$$
\Sigma_{\mathrm{ij}} q_{\mathrm{ij}}^{\prime} \log \left(p^{\prime}{ }_{1 \mathrm{i}} / p_{1 \mathrm{i}}\right)=\Sigma_{\mathrm{i}} \log \left(p^{\prime}{ }_{1 \mathrm{i}} / p_{1 \mathrm{i}}\right) \Sigma_{\mathrm{j}} q_{\mathrm{ij}}^{\prime}
$$

833 and that for each female $i$ the sum through males of the observed mating frequencies

834 involving female $i$ is, by definition, $p^{\prime}{ }_{1 \mathrm{i}}$ i.e. $\Sigma_{\mathrm{j}} q_{\mathrm{ij}}{ }_{\mathrm{ij}}=p^{\prime}{ }_{1 \mathrm{i}}$ and similarly for each male $j$ we

835 have $\Sigma_{\mathrm{i}} q_{\mathrm{ij}}^{\prime}=p_{2 \mathrm{j}}^{\prime}$. Then

836

$$
\Sigma_{\mathrm{ij}} q_{\mathrm{ij}}^{\prime} \log \left(p^{\prime}{ }_{1 \mathrm{i}} / p_{1 \mathrm{i}}\right)=\Sigma_{\mathrm{i}} \log \left(p^{\prime}{ }_{1 \mathrm{i}} / p_{1 \mathrm{i}}\right) p^{\prime}{ }_{1 \mathrm{i}}
$$

837 and

838

$$
\sum_{\mathrm{ij}} q_{\mathrm{ij}}^{\prime} \log \left(p^{\prime}{ }_{2 \mathrm{j}} / p_{2 \mathrm{j}}\right)=\Sigma_{\mathrm{j}} \log \left(p^{\prime}{ }_{2 \mathrm{j}} / p_{2 \mathrm{j}}\right) p^{\prime}{ }_{2 \mathrm{j}}
$$

839 therefore

840

$$
\Sigma_{\mathrm{ij}} \Delta\left(p^{\prime}{ }_{1 \mathrm{i}} p^{\prime}{ }_{2 \mathrm{j}}\right) \log \left(P S S_{\mathrm{ij}}\right)=
$$

841

$\Sigma_{\mathrm{i}} \log \left(p^{\prime}{ }_{1 \mathrm{i}} / p_{1 \mathrm{i}}\right) p^{\prime}{ }_{1 \mathrm{i}}-\Sigma_{\mathrm{i}} p^{\prime}{ }_{1 \mathrm{i}} \log \left(p^{\prime}{ }_{1 \mathrm{i}} / p_{1 \mathrm{i}}\right)+\Sigma_{\mathrm{j}} \log \left(p^{\prime}{ }_{2 \mathrm{j}} / p_{2 \mathrm{j}}\right) p^{\prime}{ }_{2 \mathrm{j}}-\Sigma_{\mathrm{j}} p^{\prime}{ }_{2 \mathrm{j}} \log \left(\left(p^{\prime}{ }_{2 \mathrm{i}} / p_{2 \mathrm{i}}\right)=0\right.$

842 and so the proposition is true

843

$$
E_{0}=\Sigma_{\mathrm{ij}}\left(\Delta\left(p_{1 \mathrm{i}} p_{2 \mathrm{j}}\right) \log \left(P S I_{\mathrm{ij}}\right)\right.
$$

844

845 Proposition 2

846

$$
E_{0}=D_{\mathrm{KL}}^{\prime}\left(w, q^{\prime} \| p^{\prime}\right)
$$

847 where

$$
p^{\prime}=p^{\prime}{ }_{1} p^{\prime}
$$

849

$$
w_{\mathrm{ij}}=\left(P S S_{\mathrm{ij}}-1\right) / P T I_{\mathrm{ij}}
$$




$$
D_{\mathrm{KL}}^{\prime}\left(w, q^{\prime} \| p^{\prime}\right)=\Sigma_{\mathrm{ij}} w_{\mathrm{ij}} q_{\mathrm{ij}}^{\prime} \log \left(q_{\mathrm{ij}}^{\prime} /\left(p^{\prime}{ }_{1 \mathrm{i}} p^{\prime}{ }_{\mathrm{2j}}\right)\right)
$$

851 From the model (1) and the partitions (4) and (5) in the main text we know that

854 therefore

and since $P T I_{\mathrm{ij}}=P S I_{\mathrm{ij}} \times P S S_{\mathrm{ij}}$ we obtain

and so

$$
\Delta\left(p_{1 \mathrm{i}} p_{2 \mathrm{j}}\right)=q_{\mathrm{ij}}^{\prime}\left(P S S_{\mathrm{ij}}-1\right) / P T I_{\mathrm{ij}}
$$

$$
E_{0}=\Sigma_{\mathrm{ij}}\left(\Delta\left(p_{1 \mathrm{i}} p_{2 \mathrm{j}}\right) \log \left(P S I_{\mathrm{ij}}\right)=\Sigma_{\mathrm{ij}} w_{\mathrm{ij}} q_{\mathrm{ij}}^{\prime} \log \left(P S I_{\mathrm{ij}}\right)=D_{\mathrm{KL}}^{\prime}\left(w, q^{\prime} \| p^{\prime}\right)\right.
$$

which is Kullback-Leibler-like divergence with weights $w_{\mathrm{ij}}$ in the observations $q^{\prime}$.

\section{References}

Ah-King, M., Gowaty, P. A., 2016. A conceptual review of mate choice: stochastic demography, within-sex phenotypic plasticity, and individual flexibility. Ecology and Evolution. 6, 4607-4642.

Akaike, H., Information theory and an extension of the maximum likelihood principle. In: B. N. Petrov, F. Csaki, Eds.), Second International Symposium on Information Theory, Budapest: Akademiai Kiado, 1973, pp. 267-281.

Bergstrom, C. T., Real, L. A., 2000. Toward a theory of mutual mate choice: Lessons from twosided matching. Evolutionary Ecology Research. 493-508.

Carvajal-Rodriguez, A., InfoMating. 2017, pp. Model selection and multimodel inference from mating frequency data to study sexual selection and sexual isolation effects. 
Carvajal-Rodriguez, A., Rolan-Alvarez, E., 2006. JMATING: a software for the analysis of sexual selection and sexual isolation effects from mating frequency data. BMC Evol Biol. 6, 40.

Carvajal-Rodriguez, A., Rolán-Alvarez, E., 2014. A comparative study of Gaussian mating preference functions: a key element of sympatric speciation models. Biological Journal of the Linnean Society. 113, 642-657.

Dall, S. R. X., et al., 2005. Information and its use by animals in evolutionary ecology. Trends in Ecology \& Evolution. 20, 187-193.

Darwin, C., 1871. The descent of man, and selection in relation to sex. Murray.

Edward, D. A., 2015. The description of mate choice. Behavioral Ecology. 26, 301-310.

Evren, A., Tuna, E., 2012. On some properties of goodness of fit measures based on statistical entropy. International Journal of Research and Reviews in Applied Sciences. 13, 192205.

Frank, S. A., 2009. Natural selection maximizes Fisher information. J Evol Biol. 22, 231-44.

Frank, S. A., 2012a. Natural selection. IV. The Price equation. J Evol Biol. 25, 1002-19.

Frank, S. A., 2012b. Natural selection. V. How to read the fundamental equations of evolutionary change in terms of information theory. J Evol Biol. 25, 2377-96.

Frank, S. A., 2013. Natural selection. VI. Partitioning the information in fitness and characters by path analysis. Journal of Evolutionary Biology. 26, 457-471.

Gavrilets, S., 2004. Fitness landscapes and the origin of species. Princeton University Press, Princeton, N.J.

Gibson, R. M., 1996. Female choice in sage grouse: the roles of attraction and active comparison. Behavioral Ecology and Sociobiology. 39, 55-59.

Gimelfarb, A., 1988. Processes of Pair Formation Leading to Assortative Mating in Biological Populations: Encounter-Mating Model. The American Naturalist. 131, 865-884.

Hollocher, H., et al., 1997. Incipient Speciation by Sexual Isolation in Drosophila melanogaster: Variation in Mating Preference and Correlation Between Sexes. Evolution. 51, 11751181.

Hughes, A. L., 2015. Sexual Selection and Mate Choice: Insights from Neutralist Perspectives. Evolutionary Biology. 42, 366-378.

Janicke, T., et al., 2016. Darwinian sex roles confirmed across the animal kingdom. Science Advances. 2.

Kirkpatrick, M., 1982. Sexual selection and the evolution of female choice. Evolution. 1-12.

Kuijper, B., et al., 2012. A guide to sexual selection theory. Annual Review of Ecology, Evolution, and Systematics. 43, 287-311.

Lande, R., 1981. Models of speciation by sexual selection on polygenic traits. Proceedings National Academy of Sciences, USA. 78, 3721-3725.

Pérez-Cruz, F., Kullback-Leibler divergence estimation of continuous distributions. 2008 IEEE international symposium on information theory. IEEE, 2008, pp. 1666-1670.

Pomiankowski, A., Iwasa, Y., 1998. Runaway ornament diversity caused by Fisherian sexual selection. Proceedings of the National Academy of Sciences of the United States of America. 95, 5106-5111.

Price, G. R., 1972. Extension of covariance selection mathematics. Annals of human genetics. $35,485-490$.

Prum, R. O., 2010. The Lande-Kirkpatrick mechanism is the null model of evolution by intersexual selection: implications for meaning, honesty, and design in intersexual signals. Evolution. 64, 3085-3100.

Roff, D. A., Fairbairn, D. J., 2014. The evolution of phenotypes and genetic parameters under preferential mating. Ecology and Evolution. 4, 2759-2776.

Rolán-Alvarez, E., Caballero, A., 2000. Estimating sexual selection and sexual isolation effects from mating frequencies. Evolution. 54, 30-6.

Rosenthal, G. G., 2017. Mate choice: the evolution of sexual decision making from microbes to humans. Princeton University Press. 
bioRxiv preprint doi: https://doi.org/101101/095901; this version posted January 5, 2018. The copyright holder for this preprint (which was

not certified by peer review) is the author/funder, who has granted bioRxiv a license to display the preprint in perpetuity. It is made available under aCC-BY-NC-ND 4.0 International license.

924 Roughgarden, J., et al., 2015. Sexual selection studies: a NESCent catalyst meeting. PeerJ

$925 \quad$ PrePrints 3:e680v3.

926 Schwarz, G., 1978. Estimating the dimension of a model. The Annals of Statistics. 6, 461-464.

927 Servedio, M. R., et al., 2011. Magic traits in speciation: 'magic' but not rare? Trends in Ecology

928 \& Evolution. 26, 389-397.

929 Sokal, R. R., Rohlf, F. J., 1981. Biometry. W. H. Freeman and Co., New York.

930 Wagner, G. P., 2010. The measurement theory of fitness. Evolution. 64, 1358-1376. 